\title{
ON THE NUMBER OF POSITIVE INTEGERS LESS THAN $x$ AND FREE OF PRIME DIVISORS GREATER THAN $x^{c}$
}

\section{RAMASWAMI}

Dr. Chowla recently raised the following question regarding the number of positive integers less than $x$ and free of prime divisors greater than $x^{c}$, which number is here denoted by $f(x, c)$ : For every fixed positive $c$, is $\lim \inf _{x \rightarrow \infty} f(x, c) / x>0$ ?

This paper, while incidentally answering this question in the affirmative, proves more, in fact the best ${ }^{1}$ possible result in this direction, namely:

Theorem A. A function $\phi(c)$ defined for all $c>0$ exists such that

(1) $\phi(c)>0$ and is continuous for $c>0$;

(2) for any fixed $c$

$$
f(x, c)=x \phi(c)+O(x / \log x)
$$

where the " $O$ " is uniform for $c$ greater than or equal to any given positive number.

Notation. The following symbols are used for the entities mentioned against them:

$p, p_{r}$ : any prime.

$S(x, p)$ : the set of integers less than $x$ each divisible by $p$ and free of prime divisors greater than $p$.

$T(x, p)$ : the set of integers less than $x$ each free of prime divisors greater than $p$.

$N[K]$ : the number of members of $K$, where $K$ denotes any finite set of integers.

$F(t): \sum_{p \leqq t}(1 / p)$ where $p$ runs through primes.

\section{Preliminary lemmas.}

LEMma I. For $c \geqq 1$, the theorem is true, and

$$
f(x, c)=x \phi(c)+O(1) .
$$

Proof. This is obvious. In fact, for these values of $c, \phi(c)=1$, and

$$
|f(x, c)-x \phi(c)| \leqq 1 \text {. }
$$

Lemma II. If $p_{1} \neq p_{2}$, the sets $S\left(x, p_{1}\right)$ and $S\left(x, p_{2}\right)$ are distinct, and

Received by the editors January 12, 1948, and, in revised form, July 22, 1948.

1 The " $O$ " of the theorem cannot be improved upon, as will be seen in the sequel. 
$N[S(x, p)]=N[T(x / p, p)]$.

Proof. This is obvious, from the unique factorization theorem.

LEMma III. The number of primes less than or equal to $x$ is $O(x / \log x)$ and $F(x)=\log \log x+b+O(1 / \log x)$ where $b$ is constant.

This is well known and is an "elementary theorem" in the theory of primes.

Lemma IV. If $0<c_{1} \leqq 1$, and Theorem $\mathrm{A}$ is true for $c \geqq c_{1}$, then it is true for $c \geqq c_{1} /\left(1+c_{1}\right)$.

Proof. By hypothesis $\phi(c)$ is defined for $c \geqq c_{1}$, and

$$
\phi(c)>0 \text { and is continuous for } c \geqq c_{1},
$$

(3) $f(x, c)=x \phi(c)+O(x / \log x)$ uniformly for $c_{1} \leqq c \leqq 1$,

$$
f(x, c)=x \phi(c)+O(1) \text { for } c \geqq 1, \quad \text { by Lemma } 1 \text {. }
$$

Also, obviously,

(4) $\phi(c)$ is bounded and monotonic increasing, though possibly not strictly so, for $c \geqq c_{1}$.

Let now

$$
\left.c_{2}=c /\left(1+c_{1}\right) \text { and } c_{2} \leqq d \leqq c_{1} \text { (obviously } c_{2}<c_{1}\right) .
$$

Now

$$
\begin{aligned}
f\left(x, c_{1}\right)-f(x, d)= & \sum_{x^{d}<p \leqq x^{c_{1}}} N[S(x, p)] \\
= & \sum_{x^{d}<p \leqq x^{\varepsilon_{1}}} N\left[T\left(\frac{x}{p}, p\right)\right] \\
= & \sum_{x^{d}<p \leqq x^{c_{1}}} f\left(\frac{x}{p}, \frac{\log p}{\log x-\log p}\right) \\
= & \sum_{x^{d}<p \leqq x^{c_{1}}}\left\{\frac{x}{p} \phi\left(\frac{\log p}{\log x-\log p}\right)\right\} \\
& +\sum_{p \geqq x^{1 / 2}} O(1)+\sum_{p<x^{1 / 2}} O\left(\frac{x}{p \log (x / p)}\right)
\end{aligned}
$$

by (3) and (5), since in this range

$$
\frac{\log p}{\log x-\log p}>\frac{d}{1-d} \geqq \frac{c_{2}}{1-c_{2}}=c_{1} \text {. }
$$

\section{Hence}


(6a) $f\left(x, c_{1}\right)-f(x, d)=x \int_{x^{d}}^{x^{c_{1}}} \phi\left(\frac{\log t}{\log x-\log t}\right) d F(t)+O\left(\frac{x}{\log x}\right)$

by Lemma III, where the " $O$ " is uniform with respect to $d$ in virtue of (3) and (6), and the Riemann-Stieltjes integral on the right exists in virtue of (4) and the continuity of $\phi(c)$ for $c \geqq c_{1}$. Using integration by parts for the integral and using (3) and (4) and Lemma III, we obtain (see Note 1a)

$$
f\left(x, c_{1}\right)-f(x, d)=x \int_{x^{d}}^{x^{c_{1}}} \phi\left(\frac{\log t}{\log x-\log t}\right) \frac{d t}{t \log t}+O\left(\frac{x}{\log x}\right)
$$

(uniformly for $c_{2} \leqq d \leqq c_{1}$ )

$$
=x \int_{d}^{c_{1}} \phi\left(\frac{u}{1-u}\right) \frac{d u}{u}+O\left(\frac{x}{\log x}\right)
$$

(uniformly for $c_{2} \leqq d \leqq c_{1}$ ).

This, together with (3), proves the existence of $\phi(c)$ for $c \geqq c_{1} /\left(1+c_{1}\right)$, though it is not yet clear whether $\phi(c)>0$ in $c_{1} /\left(1+c_{1}\right) \leqq c \leqq c_{1}$, and is continuous therein.

Now, let

$$
\frac{c_{1}}{1+c_{1}} \leqq d_{1}<d_{2} \leqq c_{1}
$$

Then (7) gives

$$
\begin{aligned}
& \frac{f\left(x, d_{2}\right)-f\left(x, d_{1}\right)}{x}=\int_{d_{1}}^{d_{2}} \phi\left(\frac{u}{1-u}\right) \frac{d u}{u}+O\left(\frac{1}{\log x}\right) \\
& \rightarrow \int_{d_{1}}^{d_{2}} \phi\left(\frac{u}{1-u}\right) d u \quad \text { as } x \rightarrow \infty \text {. }
\end{aligned}
$$

Hence

$$
\phi\left(d_{2}\right)-\phi\left(d_{1}\right)=\int_{d_{1}}^{d_{2}} \phi\left(\frac{u}{1-u}\right) \frac{d u}{u}>0,
$$

by (3), since in the range $d_{1}<u<d_{2}$, we have $u /(1-u)>c_{1}$.

This shows that $\phi\left(d_{2}\right) \neq 0$ for any $d_{2}$ of the kind specified in (8); for, if $\phi\left(d_{2}\right)$ were zero, then obviously $\phi\left(d_{1}\right)$ would be zero and their difference also would be so, contrary to (9). Also, obviously $\phi\left(d_{2}\right) \geqq 0$. Hence

$$
\phi(c)>0 \quad \text { for } c>c_{1} /\left(1+c_{1}\right) .
$$


Also by (9) and the hypothesis

$$
\phi(c) \text { is continuous for } c \geqq c_{1} /\left(1+c_{1}\right) \text {. }
$$

Using now the results (7), (10), and (11) and repeating the above argument with $c_{1} /\left(1+c_{1}\right)$ in place of $c_{1}$, and noting that the positiveness of $\phi\left(c_{1}\right)$ is not needed in the above argument, it follows that $\phi\left(c_{1} /\left(1+c_{1}\right)\right)>0$.

This completes the proof of the lemma.

Proof of THE THEOREM. Lemma I holds for $c \geqq 1$ and Lemma IV for $0<c<1$, if we note that the hypotheses of the lemma are satisfied for $c_{1}=1$, and hence by the result of the lemma for $c_{1}=1 / 2$ and hence by induction for $c_{1}=1 / n$ ( $n$ positive integral), and that $1 / n \rightarrow 0$ as $n \rightarrow \infty$.

Corollary. For $0<c_{1}<c_{2} \leqq 1$,

$$
\phi\left(c_{2}\right)-\phi\left(c_{1}\right)=\int_{c_{1}}^{c_{2}} \phi\left(\frac{u}{1-u}\right) \frac{d u}{u}
$$

(see also Note $1 \mathrm{~b})$.

Proof. A finite set of numbers, $d_{0}, d_{1}, d_{2}, \cdots, d_{n}$, obviously exists such that

$$
c_{1}=d_{0}<d_{1}<d_{2}<\cdots<d_{n}=c_{2}
$$

and

$$
d_{r} \geqq \frac{d_{(r+1)}}{1+d_{(r+1)}} \quad(r=0,1,2, \cdots, n-1) .
$$

To each of these intervals $\left(d_{r}, d_{r+1}\right)$ apply the result (9) and add; the corollary follows at once.

REMARKs. The " $O$ " of the theorem cannot be improved upon. This can be seen as follows:

Obviously $f(x, 1)=x+O(1)$ and

$$
f(x, 1)-f\left(x, \frac{1}{2}\right)=\sum_{x^{1 / 2}<p \leqq x}\left[\frac{x}{p}\right]=x \sum_{x^{1 / 2}<p \leqq x}\left(\frac{1}{p}\right)-G(x)
$$

where $G(x)=\sum_{x^{1 / 2}<p \leqq x}\{x / p\}$ and $\{y\}$ denotes fractional part of $y$.

Hence $f(x, 1 / 2)=x(1-\log 2)+o(x / \log x)+G(x)$ by the prime number theory (see Note 1c). But $G(x)>k x / \log x$ where $k$ is a fixed positive number, as can be easily seen from the prime number theory.

Note 1. (a) The deduction of (7) from (6a) is based on the following: 
We observe that the Riemann-Stieltjes integral

$$
\begin{aligned}
\int_{x^{d}}^{x^{c_{1}}} \phi\left(\frac{\log t}{\log x-\log t}\right) d F(t)= & {\left[F(t) \phi\left(\frac{\log t}{\log x-\log t}\right)\right]_{x^{d}}^{x^{c_{1}}} } \\
& -\int_{x^{d}}^{x^{c_{1}}} F(t) d \phi \\
= & {\left[(\log \log t+b) \phi\left(\frac{\log t}{\log x-\log t}\right)\right]_{x^{d}}^{x^{c_{1}}} } \\
& -\int_{x^{d}}^{x^{c_{1}}}(\log \log t+b) d \phi+R
\end{aligned}
$$

which, by the substitution $t=x^{u}$ performed after integration by parts of the integral on the right, equals

$$
\int_{d}^{c_{1}} \phi\left(\frac{u}{1-u}\right) \frac{d u}{u}+R
$$

where, by Lemma III,

$$
R=O\left(\frac{1}{d \log x}\right)-O\left(\frac{1}{d \log x}\right)-\int_{x^{d}}^{x^{c_{1}}} O\left(\frac{1}{\log t}\right) d \phi
$$

where, on account of (4), the integral also is $O\left(d^{-1} \log ^{-1} x\right)$.

(b) The integral equation for $\phi(c)$ may be used for successive computation of the function. Starting with $\phi(c)=1$ for $c \geqq 1$, one observes that $c /(1-c) \geqq 1$ for $1 / 2 \leqq c<1$ so that

$$
\phi(c)=1-\int_{c}^{1} \frac{d u}{u}=1+\log c \quad \text { for } 1 / 2 \leqq c \leqq 1 .
$$

This result can be used to compute $\phi(c)$ on the interval $(1 / 3,1 / 2)$ and so on; and an easy induction shows that

$$
\phi(c)=1+\sum_{r=1}^{\infty}(-1)^{r} \psi_{r}(c), \quad c>0,
$$

where $\psi_{1}(c)=\int_{c}^{1} d u / u$ for $c \leqq 1$, and $\psi_{1}(c)=0$ for $c>1$, and

$$
\psi_{r}(c)=\int_{0}^{1} \psi_{r-1}\left(\frac{u}{1-u}\right) \frac{d u}{u}, \quad \text { for } c>0 .
$$

One notes that $\psi_{r}(c)=0$ for $c \geqq 1 / r$ (so that the infinite series is actually a finite sum) and also that the functions $\psi_{r}(c), r \geqq 2$, are not elementary functions. 
(c) From the known result $\pi(x)=x / \log x+x / \log ^{2} x+o\left(x / \log ^{2} x\right)$ follows

$$
\begin{aligned}
\sum_{x^{1 / 2}<p \leqq x} \frac{1}{p}= & \int_{x^{1 / 2}}^{x} \frac{1}{t} d \pi(t)=\frac{\pi(x)}{x}-\frac{\pi\left(x^{1 / 2}\right)}{x^{1 / 2}}+\int_{x^{1 / 2}}^{x} \frac{\pi(t)}{t^{2}} d t \\
= & -\frac{1}{\log x}+o\left(\frac{1}{\log x}\right) \\
& +\int_{x^{1 / 2}}^{x}\left[\frac{t}{\log t}+\frac{t}{\log ^{2} t}+o\left(\frac{t}{\log ^{2} t}\right)\right] \frac{d t}{t^{2}} \\
= & \log 2+o\left(\frac{1}{\log x}\right) .
\end{aligned}
$$

Note 2. From the corollary to the theorem follows, for $0<c_{1}<c_{2}$ $\leqq c_{1} /\left(1-c_{1}\right)$ and $c_{2} \leqq 1$,

$$
\phi\left(c_{2}\right)-\phi\left(c_{1}\right) \geqq \phi\left(c_{2}\right) \log c_{2} / c_{1}>\phi\left(c_{2}\right)\left(1-c_{1} / c_{2}\right)
$$

from which follows $\phi\left(c_{2}\right) / c_{2}>\phi\left(c_{1}\right) / c_{1}$, whence, arguing as in the proof of the corollary, one sees that $\phi(c) / c$ is a strictly monotonic increasing function in $0<c \leqq 1$.

$\phi(c)$ has other interesting properties, which will be published shortly. One such is that for every fixed $n, \phi(c) / c^{n} \rightarrow 0$ as $c \rightarrow+0$. This result, together with the now obvious result

$$
\lim _{x \rightarrow \infty} \frac{f(x, c)}{x}=\phi(c)>0, \quad c \text { fixed and positive, }
$$

was communicated to Dr. Chowla in August of 1947. I understand from his reply that Vijayaraghavan already was in possession of a proof of his (Chowla's) conjecture that

$$
\liminf _{x \rightarrow \infty} \frac{f(x, c)}{x}>0, \quad \text { for } c \text { positive and fixed. }
$$

In conclusion, I wish to thank the referees for their suggestions which have led to the clarification and additions of content contained in Note 1.

ANDHRA UNIVERSITY 